be tested. Says Peter Meldrum, the president and CEO of Myriad Genetics, who cofounded the company in 1991 with Mark Skolnick (a codiscoverer of the $B R C A 1$ gene), "we will be offering our test to women at high risk of developing breast cancer." Although Meldrum says that Myriad Genetics will not be offering prenatal testing or testing of minors, he admits that if a woman without a family history of the disease wants to be tested - with the support of her physician she will not be denied that opportunity. "We cannot be paternalistic," he says.

Most parties agree that genetic testing for breast cancer needs to be performed within academic centers using Institutional Review Board-approved protocols to assess the sensitivity, specificity and predictive value of the $B R C A 1$ tests. Ensuring that all commercial centers offer- ing genetic testing follow established protocols might prove difficult, because they do not need the approval of the US Food and Drug Administration. Collins sees the solution in a massive expansion of the number of research protocols at academic centers in which women could enroll to obtain the BRCA1 test while also receiving essential education and counseling. Meldrum emphasizes that women will not receive the Myriad Genetics test unless they sign a consent form stating that they have read and understood the educational material provided and have also received counseling. "Educating not only patients but health care providers about the benefits and limitations of testing for $B R C A 1$ mutations is essential," says Wolff.

Women testing positive for a BRCA1 mutation have several choices in an attempt to prevent breast cancer. However, "there are little data indicating which is the best option for women with a positive test result," says Holtzman, "Bilateral mastectomy, frequent monitoring, prophylactic chemotherapy or mammography screening at a younger age need to be compared in a systematic manner, including randomized clinical trials."

"Yet ah! why should they know their fate? ... where ignorance is bliss, 'tis folly to be wise." The prophetic and oft' misquoted words of Thomas Gray, the 18th-century American poet, hold part of the answer to the current dilemma. Is genetic predisposition a situation where ignorance is bliss? The advent of genetic testing has now made "ignorance" an option, but an option that, paradoxically, only the well-informed woman can choose.

ORLA SMITH

\title{
Making geneticists out of physicians
}

Whether it is a sign of the times or a watershed in health care, all doctors may soon be required to demonstrate proficiency in interpreting genetic tests and explaining the results to their patients. A Task Force on Genetic Testing, jointly sponsored by the US National Institutes of Health (NIH) and the Department of Energy (DOE), which met last month in Baltimore, says genetics needs to become an essential element of medical practice.

The task force's opinion reflects growing consensus among medical policy makers that physicians, regardless of specialty, will confront genetic testing at some point in their careers.

"We think [all physicians] should know something about genetics," said Neil Holtzman, the task force chairman. "To ensure that they had it, they would need some demonstration of competency in genetics and genetic testing for licensure or relicensure."

However, Holtzman, who is also director of the Johns Hopkins Medical Institutions' center for Genetics and Public Policy Studies, cautioned that the licensing issue is still in its infancy. "We're just beginning to discuss" it, he said. Even so, he said, "Somewhat surprisingly, the task force seemed to be in agreement."

The task force was established in 1994 by the NIH-DOE Working Group on Ethical, Legal, and Social Implications of Human Genome Research, or ELSI, and consists of 15 voting members from the private and public sectors, including insurance industry, biotechnology and professional society representatives. In addition, there are five non-voting government representatives on the committee.

Holtzman said the recommendation that physicians be required to demonstrate competency in genetics and genetic testing - a yet-to-be defined standard - was supported by the American Medical Association's (AMA) representative to the task force, Patricia Numann. In addition, Myron Genel, another member of the AMA's Council on Scientific Affairs, agreed with the recommendation. "The explosion of knowledge is such that there are certainly not enough geneticists or genetics counselors" to handle the growing interest of patients in the technology, Genel says. "Physicians are going to need to become more versed in the applications and limitations of genetic testing."

Genel, who is also a professor of pediatrics and associate dean at the Yale University School of Medicine, indicated that the position taken by the AMA at the Baltimore meeting was not the official stance of the association at large, and would not be put to a vote until next December, at the earliest. However, he said the council has been considering the implications of genetics for AMA members for some time.

Holtzman was involved in a 1991 survey of selected primary care physicians and psychiatrists to determine how well-versed these doctors were in genetics. The study, published in the August 1993 edition of Academic Medicine, found that those doctors who responded answered fewer than three-quarters of the genetics questions correctly. Professional geneticists in the survey, by comparison, answered nearly 95 percent of those questions correctly.

Thus, the study's authors concluded, "curriculum planners should ensure that instruction in genetics (including relevant skills such as the interpretation of probabilistic results and their explanation to patients) is integrated throughout all years of medical school and is provided to residents in primary care."

Janet Hafler, associate director of faculty development at Harvard Medical School, said that should the interim principles eventually become official guidelines, Harvard would adapt. "Schools do change to abide by guidelines," said Hafler, who noted that Harvard and other institutions moved swiftly to incorporate HIV into their core curricula.

Holtzman said the task force will convene again in September, and should have a final report by the end of this year. At that time, were the report to include some incarnation of a competency requirement, implementation could require action by such bodies as the Food and Drug Administration, state or federal legislators, and professional societies.

ADAM MARCUS Baltimore, Maryland 